\title{
Simulation of MIMO System with STBC in Simulink and MATLAB
}

\author{
Swapnil T. Patil \\ Department of Electronics and Telecommunication \\ Sinhgad Academy of Engineering, Kondhwa \\ Pune, India
}

\author{
Pratap N. Shinde \\ Department of Electronics and Telecommunication \\ Sinhgad Academy of Engineering, Kondhwa \\ Pune, India
}

\begin{abstract}
The space-time block coding (STBC) makes use of a high algebraic structure to provide diversity gain. Paper shows that the multiple receiver antennas increase the diversity, which results in high SNR. The two illustrative examples of Multiple Input Multiple Output (MIMO) system in wireless network using STBC are presented. Firstly, paper compares the case of single user while incorporating multiple receiving antennas. Secondly, paper compares the case of multiple users while incorporating multiple receiving antennas. The simulation results after experimental testing are in good agreement.
\end{abstract}

\section{General Terms}

Wireless communications, MIMO system, Space-time block coding (STBC).

\section{Keywords}

MIMO system, orthogonal design, diversity, space-time block code (STBC).

\section{INTRODUCTION}

Extensive computer simulations of any complex hardware are performed on various software prior practical implementations. Wireless Communication system e.g. mobile communication, radar etc. are simulated with researchintensive models of hardware and underlying mathematics [1]. In simulation all aspects are taken into account (hardware and software). The main idea is to be able to observe directly effects of component design or architecture on performance of the system and optimization of components. The main focus is on system performance which is in the form of Quality-ofService (QoS) or in bit-error-rate (BER).

As we know that wireless spectrum used in wireless communication is very expensive thus we need to design system with more capacity and less requirement of Bandwidth. Therefore there is need to optimize communication system with some techniques which gives high network capacity without increasing bandwidth. To optimize communication system interference cancellation is important to enhance the network capacity. In propagation media due to destructive addition of multipath signals and interference from other user's wireless communication suffers attenuation. Channel conditions are usually Rayleigh in nature and it makes receiver difficult to determine the transmitted signal. If receiver is provided with less attenuated replica of the original signal, receiver now will have many options to predict (recover) original data. This technique of providing multiple copies is known as diversity [2]. This is the best technique which achieves faithful transmission over a fading channel. The main motivation of diversity is to recover robust replica of transmitted signal at receiver side by combining multiple copies which are independently faded. By interference cancellation in Time Division Multiple Access (TDMA) and Code Division Multiple Access (CDMA) mobile users can potentially allow to share same time slots and same code words.

In this paper, we considered multiple antenna scenarios at both receiver and transmitter side. In paper [3], the multiuser multi-antenna interference cancellation technique is addressed for first time. In this paper, Tarokh et al. consider the cases where $\mathrm{N}$ transmitter antennas are equipped to each user. They have shown that each user can be decoded separately with antennas equal to $\mathrm{N}$ times number of users. In the work by Naguib et al [4]. They have used orthogonal space time block code (STBC) properties and provided an interference cancellation method using very less receive antennas equal to number of users. But this method works for only users with two transmitting antennas. The work in [5] improved the system to more number of antennas but only for two users. In [6], Javad et al. showed why the work in [4] cannot be applied to more number of transmit antennas when the constellation used is complex. They offered an alternative method based on similar modulation scheme of Quasi Orthogonal Space Time Block Code (QOSTBC) [7-8]. They also used number of receiver antennas equal to number of users.

When multiple antennas are available then the Space-time codes can be designed so as to provide highest possible diversity. But on the other hand, using multiple-antenna system codes like BLAST [9] can achieve potentially high data rate but with less diversity than space-time codes[10]. Codes like Golden [11] and Perfect [12] can achieve both full diversity and at the same time can provide high data rate. But drawback of these codes is that they require receiving antennas equal to number of transmitting antennas, because they use sphere decoding. On the other hand orthogonal space-time block code (OSTBC) and Quasi orthogonal spacetime block code (QOSTBC) [13] achieve full diversity and require less receive antennas $m=k$. So codes OSTBC and QOSTBC are advantageous to use in our simulation.

The outline of the paper follows next. In Section II, we describe a MIMO system and its features. We provide the details of space time block coding and described channel model in Section III. Section IV provides simulation results and section $\mathrm{V}$ concludes the paper. 
Transmitter

Receiver

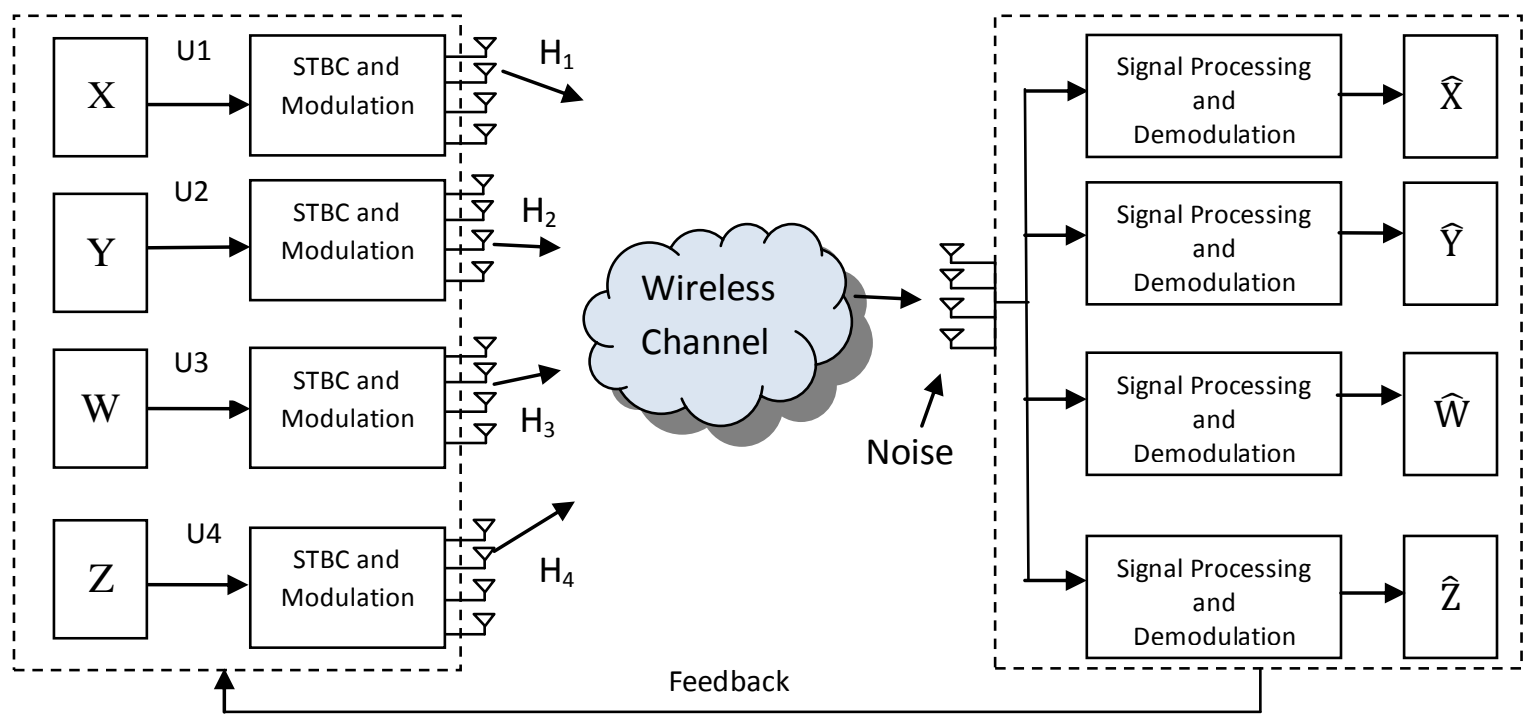

Fig.1. STBC coded multiuser MIMO system

\section{WIRELESS COMMUNCATION USING MIMO}

In the last two decade or so, there has been huge growth and development in the wireless communication market. At the starting there was focus on only speech processing and the implementation challenge was to increase number of users per spectrum and in given bandwidth [1]. Today there is a very huge demand of high data rates, for various advanced internet services like video calling, net browsing, navigation, high definition Tele-Vision etc and this demand is increasing day by day. To meet this growing demand various spectrally efficient protocols have been designed. Previous studies on MIMO have shown that if $n$ transmit antennas and $m$ receive antennas are equipped then capacity (data rate) of the system is improved up to a factor of $\mathrm{n}$ or $\mathrm{m}$, based on wireless channel conditions. The main obstacles in wireless communication are scattering, multipath, attenuation and Doppler shift [1]. Together we can call or refer them as noise in system. We can use spacial multiplexing to increase much of the system capacity. Diversity concept with spatially separated antennas can be used to increase system capacity. We can take advantage of fading i.e. every signal traverse different path, fades independently and meet at transmitter. Probability of getting correct data is high when we have more than one observation because receiver gets more number of copies to determine original data. Space Time coding [14] is best approach for achieving spacial diversity, without knowledge of channel state information (CSI) at transmitter.

MIMO can be sub divided into three main parts precoding, spacial multiplexing and diversity coding [18].

\subsection{Precoding}

Precoding in short can be defined as multi-stream beamforming. In this case all spacial processing is performed at transmitting side. Same signal is transmitted from number of antennas with appropriate phase and gain, so that receiver input signal power is maximized. Benefits of this are increased signal gain achieved by constructive addition of signals transmitted by multiple antennas and decreased multipath fading effects. But to use precoding at both transmitter and receiver knowledge of channel state information is needed.

\subsection{Spacial Multiplexing}

In Spacial Multiplexing (SM) multiple lower rate signal streams are created form a high rate signal and using same frequency channel each signal stream is transmitted through a different transmit antenna. This technique is very useful as it increases channel capacity at high SNR. This method can be used without channel state information (CSI) at transmitter. If CSI is available then we can take advantage of precoding.

\subsection{Diversity}

Diversity coding technique is used when there is no knowledge of channel state information at transmitter. Unlike multiple streams in SM, here single stream is transmitted but space-time coding technique is used. This technique uses orthogonality for signal transmission. Diversity coding does not use array gain or beamforming as there is no channel knowledge. If channel state information is present at transmitter then spacial multiplexing can be combined with diversity coding.

\section{SPACE TIME BLOCK CODES AND CHANNEL MODEL}

Since the evolution of Space Time Block Code (STBC), they have been of wide interest because they provide significant increase in transmission rate, range and reliability. More interesting is that we get these benefits without need of increase in bandwidth. By using rich structure of STBC we can reduce number of required receive antennas to number of users and provide diversity of NM [12] and suppress k-1 co channel space time users. The Main idea of using Multiple Input Multiple Output system is ability to turn multipath propagation, which is usually an obstacle in conventional wireless communication system into benefit.

In our first experiment we used a wireless communication system with two antennas at transmitter $\mathrm{n}_{1}$ and $\mathrm{n}_{2}$ and assumed the channel to be flat fading. Let $h_{i j}$ be path gains from transmit antenna $i$ to receive antenna $\mathrm{j}$. Symbols to be transmitted are $s_{1}, s_{2}, s_{3}, s_{4}, s_{5}, s_{6} \ldots$ then transmission can be expressed in matrix form as 


$$
s=\left[\begin{array}{cc}
S_{1} & S_{2} \\
-S_{2}{ }^{*} & S_{1}{ }^{*}
\end{array}\right]
$$

Columns of the matrix represent number of antennas and rows represents time period. Therefore at time $t_{1}$, symbols from first row $\mathrm{s} 1$ and $\mathrm{s} 2$ are sent form antenna $\mathrm{n} 1$ and $\mathrm{n} 2$ respectively. At time $\mathrm{t}_{2}$ symbols form second row $-\mathrm{s} 2^{*}$ and $\mathrm{s} 1^{*}$ are sent from antenna $\mathrm{n} 1$ and $\mathrm{n} 2$ we will plot in Table 1 for better understanding.

Now ignoring the noise, corresponding received signal will be as shown in Table 2 . $h 1$ is channel coefficient form transmit antenna $\mathrm{n}_{1}$ to receive antenna $\mathrm{m}_{1}$ and $\mathrm{h} 2$ is channel coefficient from antenna $n_{2}$ to $m_{1}$, receive signal can also be written in the form

$$
\left[\begin{array}{c}
r_{1} \\
r_{2}{ }^{*}
\end{array}\right]=\left[\begin{array}{cc}
h_{1} & h_{2} \\
h_{2}{ }^{*} & -h_{1}{ }^{*}
\end{array}\right]\left[\begin{array}{l}
s_{1} \\
s_{2}
\end{array}\right], r=H s
$$

To make this method more general, we extended this to $\mathrm{n}$ transmitting antennas and $\mathrm{m}$ receiving antennas, signal $r_{t}^{j}$ represents received signal by antenna $j$ at time $t$, and written as

$$
r_{t}^{j}=\sum_{i=1}^{n} h_{i j} s_{t}^{j}+n_{t}^{j}
$$

Where $n_{t}^{j}$ is noise received at antenna $\mathrm{j}$ at time $\mathrm{t}$, and it is considered as independent, complex, zero mean Gaussian random variable. $1 / n$ is average energy of symbols transmitted by each $\mathrm{n}$ antenna. Assuming there is perfect channel knowledge $h_{i j}$ (from transmit antenna i to receive antenna $j$ ) at receiver side, the decision metric was

$$
\sum_{t=1}^{l} \sum_{j=1}^{m}\left|r_{t}^{j}-\sum_{i=1}^{n} h_{i j} S_{t}^{j}\right|^{2}
$$

Over

all code

words $s_{1}^{1} s_{1}^{2} \ldots s_{1}^{n} s_{2}^{1} s_{2}^{2} \ldots s_{2}^{n} \ldots \ldots s_{l}^{1} s_{l}^{2} \ldots s_{l}^{n}$, (where $\mathrm{n} \quad$ is number of receiving antennas and $l$ length of frame, decision is taken in favor of the codeword that minimizes this sum).

For real symbol constellation, matrix of size $\mathrm{n}$ with orthogonal symbols $\underline{ \pm} s_{1}, \underline{+} s_{2}, \ldots \pm s_{n}$ can be written for 4 transmit antenna as

$$
S=\left[\begin{array}{cccr}
s_{1} & S_{2} & S_{3} & S_{4} \\
-S_{2} & S_{1} & -S_{4} & S_{3} \\
-S_{3} & S_{4} & S_{1} & -s_{2} \\
-S_{4} & -S_{3} & S_{2} & S_{1}
\end{array}\right]
$$

At first time slot first row $\left(s_{1}, S_{2}, s_{3}, s_{4}\right)$ is transmitted by four transmit antennas and at second time slot second row is transmitted $\left(-s_{2}, s_{1},-s_{4}, s_{3}\right)$ and so on.

Design provides transmit diversity by using orthogonal design, this provides maximum possible rate and can achieve full diversity so there is no loss in bandwidth. Because of orthogonal design we used simple maximum likelihood decoding algorithm.
Table 1: Block codes for two transmit antennas

\begin{tabular}{|c|c|c|}
\hline Time & Antenna $\mathbf{n}_{\mathbf{1}}$ & Antenna $\mathbf{n}_{\mathbf{2}}$ \\
\hline $\mathrm{t}$ & $\mathrm{s}_{1}$ & $\mathrm{~s}_{2}$ \\
\hline $\mathrm{t}+\mathrm{T}$ & $-\mathrm{s}_{2}{ }^{*}$ & $\mathrm{~s}_{1}{ }^{*}$ \\
\hline $\mathrm{t}+2 \mathrm{~T}$ & $\mathrm{~s}_{3}$ & $\mathrm{~s}_{4}$ \\
\hline $\mathrm{t}+3 \mathrm{~T}$ & $-\mathrm{s}_{4}{ }^{*}$ & $\mathrm{~s}_{3}{ }^{*}$ \\
\hline $\mathrm{t}+4 \mathrm{~T}$ & $\mathrm{~s}_{5}$ & $\mathrm{~s}_{6}$ \\
\hline $\mathrm{t}+5 \mathrm{~T}$ & $-\mathrm{s}_{6}{ }^{*}$ & $\mathrm{~s}_{5}{ }^{*}$ \\
\hline & $\cdots$ & $\cdots$ \\
\hline
\end{tabular}

Table 2: Received codes at receiver

\begin{tabular}{|c|c|}
\hline Time & Antenna $\mathrm{m}_{\mathbf{1}}$ \\
\hline $\mathrm{t}$ & $\mathrm{h}_{1} \mathrm{~s}_{1}+\mathrm{h}_{2} \mathrm{~s}_{2}$ \\
\hline $\mathrm{t}+\mathrm{T}$ & $-\mathrm{h}_{1} \mathrm{~s}_{2}{ }^{*}+\mathrm{h}_{2} \mathrm{~s}_{1}{ }^{*}$ \\
\hline $\mathrm{t}+2 \mathrm{~T}$ & $\mathrm{~h}_{1} \mathrm{~s}_{3}+\mathrm{h}_{2} \mathrm{~s}_{4}$ \\
\hline $\mathrm{t}+3 \mathrm{~T}$ & $-\mathrm{h}_{1} \mathrm{~s}_{4}{ }^{*}+\mathrm{h}_{2} \mathrm{~s}_{3}{ }^{*}$ \\
\hline & $\cdots$ \\
\hline
\end{tabular}

\section{SIMULATION RESULTS}

In this section we have provided simulation results for single user and multiple users. Figure 1 illustrates the system block diagram. We took two examples, one with single user and other with multiuser (four users).

Experiment 1: We considered single user with two transmitting antennas and observed results by increasing number of receiving antennas. We plotted these results for better understanding as shown in Figure 2. We used QPSK modulation and space-time block coding (STBC) for transmission and reception of symbol. To prepare Simulation model we took source as Binary Generator and signal was then modulated using QPSK modulator. Orthogonal spacetime block code (OSTBC) was used for transmission of signal. We used two antennas at transmitter and signals were passed through Rayleigh fading channel, AWGN was used to add white Gaussian noise to input signal. This signal was then received by OSTBC decoder and finally we demodulated the signal using QPSK demodulator to predict original signal. Comparing the output signal and input signal we plotted the graph of signal to noise ratio (SNR) versus Bit error rate (BER).

We followed this method for one, two and four receive antennas and compared their results in a single graph.

Experiment 2: As per system block diagram Figure 1 we modeled simulation setup for multiuser MIMO system. We took integer generator and modulated the signal by 4-QAM, using orthogonal space-time block coding (OSTBC) and four transmitting antennas for each user. Taking into consideration channel state information (CSI) we multiplied signal with precoder. Signal traversed channel and AWGN noise was added. OSTBC combiner was used to receive the signal and simple maximum likelihood (ML) decoding was used. We demodulated the signal and compared it with original transmitted signal. 


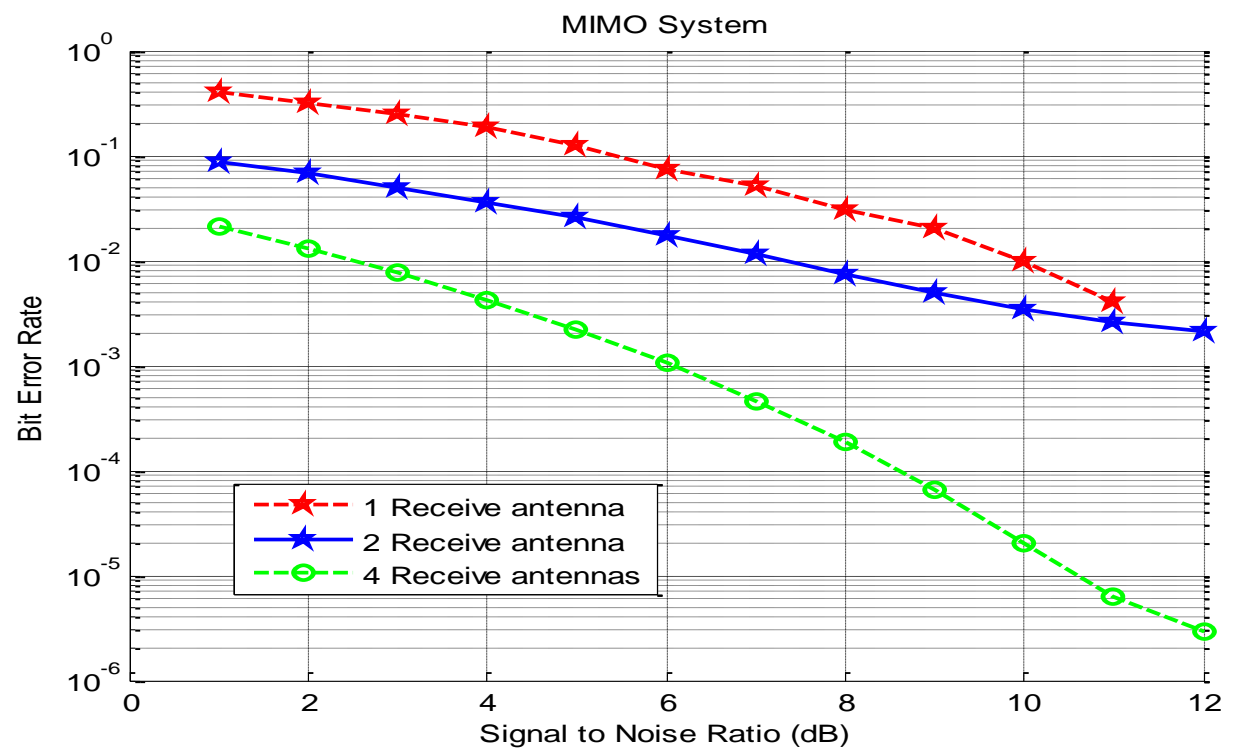

Figure 2. Single user MIMO STBC system with two transmitting antennas and QPSK modulation

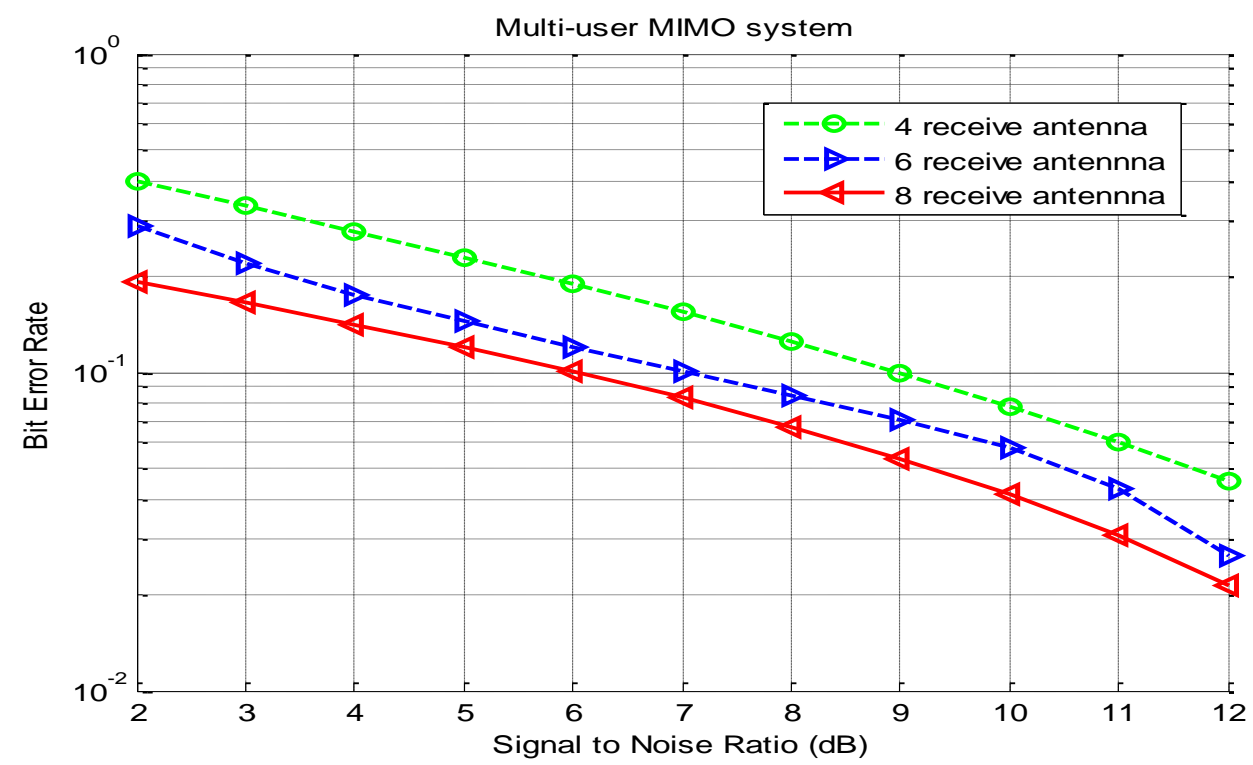

Figure 3. Four users MIMO with four transmitting antennas for each user and QAM modulation

We followed this procedure for four; six and eight receive antennas and plot the results for Signal to Noise Ratio (SNR) versus bit error rate (BER).

\section{CONCLUSION}

Paper showed how the space time block codes (STBC) using multiple receive antennas increases the diversity, which results in high (Signal to Noise Ratio) SNR. It is observed that by using multiple receive antennas SNR increases for both single and multiple users.

Future scope of this idea includes examination of the results for more than 8 receive antennas for multiple users.

\section{REFERENCES}

[1] M. Viberg, T. Boman, U. Carlberg, L. Pettersson, S. Ali, E. Arabi, M. Bilal and O. Moussa, "Simulation of MIMO Antenna Systems in Simulink and Embedded Matlab".

[2] V. Tarokh, H. Jafarkhani, and A. R. Calderbank, "Spacetime block coding for wireless communication: Performance result" IEEE Journal on selected areas in communication vol.17, no. 3, March 1999.

[3] V. Tarokh, A. Naguib, N. Seshadri, and A. R. Calderbank, "Combined array processing and space-time coding," IEEE Trans. Inform. Theory,vol. 45. pp. 11211128, May 1999. 
[4] A. F. Naguib, N. Seshadri, and A. R. Calderbank, "Applications of space-time block codes and interference suppression for high capacity and high data rate wireless systems," in Proc. 32nd Asilomar Conf. Signals, Systems and Computers, pp. 1803-1810, 1998.

[5] N. Al-Dhahir and A. R. Calderbank, "Further results on interference cancellation and space-time block codes," in Proc. 35th Asilomar conf. on Signals, Systems and Computers, pp. 257-262, Oct. 2001.

[6] J. Kazemitabar and H. Jafarkhani, "Multiuser interference cancellation and detection for users with more than two transmit antennas," IEEE Trans. Commun., vol. 56, no. 4, pp. 574-583, Apr. 2008.

[7] H. Jafarkhani, "A quasi-orthogonal space-time blcok code," IEEE Trans. Commun., vol 49, no. 1, Jan. 2001.

[8] W. Su and X. Xia, "Quasi-orthogonal space-time block codes with full diversity," IEEE Trans. Inform. Theory, vol. 50, no. 10, pp. 2331-2347, Nov. 2004.

[9] P. W. Wolniansky, G. J. Foschini, G. D. Golden, and R. A. Valenzuela, "V-BLAST: an architecture for realizing very high data rates over the rich-scattering wireless channel," invited paper, in Proc. ISSSE-98, Pisa, Italy, Sept. 1998.

[10] L. Zheng and D. Tse, "Diversity and multiplexing: a fundamental tradeoff in multiple-antenna channels," IEEE Trans. Inform. Theory, vol. 49, no. 5, May 2003.
[11] J.-C. Belfiore, G. Rekaya, and E. Viterbo, "The Golden Code: a $2 \times 2$ full-rate space-time code with nonvanishing determinants," IEEE Trans.Inform. Theory, vol. 51, no. 4, Apr. 2005.

[12] F. E. Oggier, G. Rekaya, J.-C. Belfiore, and E. Viterbo, "Perfect spacetime block codes," IEEE Trans. Inform. Theory, vol. 52, no. 9, Sept. 2006.

[13] Feng Li, Student Member, IEEE, and Hamid Jafarkhani, Fellow, IEEE, "Interference Cancellation and Detection for More than Two Users", IEEE transactions on communications, vol. 59, no. 3, march 2011.

[14] V. Tarokh, H. Jafarkhani, and A. R. Calderbank, "Spacetime block codes from orthogonal designs," IEEE Trans. Inform. Theory, vol. 45, pp. 1456-1467, July 1999.

[15] G. Ganesan and P. Stoica, "Space-time Block Codes: A Maximum SNR Approach", IEEE Trans. Inform. Theory, Vol. 47, No. 4, pp. 1650-1656, 2001

[16] Liang Xian and Huaping Liu, "Rate-One Space-Time Block Codes With Full Diversity", IEEE Trans. Commun, Vol. 53 , No. 12, Dec. 2005.

[17] W. Su and X.-G. Xia, "Two Generalized Complex Orthogonal Space-time Block Codes of Rates 7/11 and $3 / 5$ for 5 and 6 Transmit Antennas", presented as recent results in the International Symposium on Information Theory (ISIT'01), Washington D.C., June 2001. To appear in IEEE Trans. Inform. Theory.

[18] "http://en.wikipedia.org/wiki/MIMO". 